\title{
S2OSC: A Holistic Semi-Supervised Approach for Open Set Classification
}

\author{
Yang Yang * \\ Nanjing University of Science and Technology \\ yyang@njust.edu.cn
}

\author{
Zhen-Qiang Sun \\ Nanjing Normal University \\ enderman19980125@outlook.com
}

\author{
Jian Yang \\ Nanjing University of Science and Technology \\ csjyang@njust.edu.cn
}

\author{
Hui Xiong \\ Rutgers University \\ hxiong@rutgers .edu
}

\begin{abstract}
Open set classification (OSC) tackles the problem of determining whether the data are in-class or out-of-class during inference, when only provided with a set of in-class examples at training time. Traditional OSC methods usually train discriminative or generative models with in-class data, then utilize the pre-trained models to classify test data directly. However, these methods always suffer from embedding confusion problem, i.e., partial out-of-class instances are mixed with in-class ones of similar semantics, making it difficult to classify. To solve this problem, we unify semi-supervised learning to develop a novel OSC algorithm, S2OSC, that incorporates out-of-class instances filtering and model re-training in a transductive manner. In detail, given a pool of newly coming test data, S2OSC firstly filters distinct out-of-class instances using the pre-trained model, and annotates super-class for them. Then, S2OSC trains a holistic classification model by combing in-class and out-of-class labeled data and remaining unlabeled test data in semi-supervised paradigm, which also integrates pre-trained model for knowledge distillation to further separate mixed instances. Despite its simplicity, the experimental results show that S2OSC achieves state-of-the-art performance across a variety of OSC tasks, including $85.4 \%$ of F1 on CIFAR-10 with only 300 pseudo-labels. We also demonstrate how S2OSC can be expanded to incremental OSC setting effectively with streaming data.
\end{abstract}

\section{Introduction}

The real-world is changing dynamically, and many applications are non-stationary, which always receive the data containing out-of-class (also called unknown class) instances, for example, selfdriving cars need to identify unknown objects, face recognition system needs to distinguish unseen personal pictures, image retrieval often emerges new categories, etc. This problem is defined as "Open Set Classification (OSC)" in literature [1]. Different from traditional Closed Set Classification (CSC) which assumes training and testing data are draw from same spaces, i.e., the label and feature spaces, OSC aims to not only accurately classify in-class (also called known class) instances, but also effectively detect out-of-class instances. Besides, a generalized situation is that out-of-class instances will arise continuously with the streaming data, i.e., unknown classes appear incrementally, which is also defined as incremental OSC. China.

*Yang Yang and Jian Yang are with the Nanjing University of Science and Technology, Nanjing 210094, 
Both anomaly detection $[2,3]$ and zero-shot learning $[4,5]$ are related to open set classification. They have similar objectives to detect anomaly/out-of-class instances given a set of in-class examples. In contrast, anomaly detection (also called outlier detection) is an unsupervised learning task [3]. The goal is to separate abnormal in-class instances from normal ones, where the distinction from OSC is that differences between unknown and known classes are larger than that between anomalies and known classes [6]. Unlike anomaly detection, zero-shot learning (ZSL) focuses on constructing related OSC models to address unknown class detection issue, which merely utilize in-class examples and semantic information about unknown classes. Whereas the standard ZSL methods only test out-of-class instances, rather than test both known and unknown classes. Thus, generalized zero-shot learning (GZSL) is proposed, which automatically detect known and unknown classes simultaneously. For example, $[4,5]$ learned more reliable classification models by measuring the distance between examples and corresponding in-class/out-of-class semantic embeddings. However, both ZSL and GZSL assume that semantic information (for example, attributes or descriptions) of the unknown classes is given, which is limited to classify with prior knowledge.

Therefore, a more realistic classification is to detect out-of-class without any information of unknown classes. With the advent of deep learning, recent OSC approaches can mainly be divided into two aspects: discriminative and generative models. Discriminative models mainly utilize the powerful feature learning and prediction capabilities of deep models to design corresponding distance or prediction confidence measures [7,8]. In contrast, generative models mainly employ the adversarial learning to generate out-of-class instances near the decision margin that can fool the discriminative model $[9,10]$. In summary, existing OSC approaches focus on learning a representative latent space for in-class examples that preserves details of the given classes. In this case, it is assumed that when presents out-of-class instances to the pre-trained network, it will generate a poor embedding that reports a relatively higher classification error. However, this assumption does not hold for all situations. For example, as shown in Figure 1, experiments on MNIST suggest that networks (discriminative and generative models $[8,11]$ ) trained with simple content have high novelty detection accuracy, i.e., the embeddings of out-of-class digits 5 and 6 are well separated from in-class examples. In contrast, instances with complex content, such as CIFAR-10, have much low novelty detection accuracy. This is because latent embeddings learned for in-class examples can also inherently apply to represent some out-of-class instances, for example, the latent embeddings learned for cat (number 3 in Figure 1 (b) and (d)) are also able to represent some instances of other out-of-class animal such as $\operatorname{dog}$ (number 5 in Figure 1 (b) and (d)), considering similar appearance, color and other information. This phenomenon is defined as Embedding Confusion in this paper.

We note that out-of-class instances always include confused and distinct ones. Thereby we can firstly select the distinct out-of-class instances, then re-train a new classifier by combing stored in-class examples in semi-supervised paradigm. Meanwhile, the pre-trained model can be employed as a teacher model, which not only ensures that in-class instances are well represented, but also guarantees that out-of-class instances are poorly represented. In result, the learned classifier can obtain well separated embeddings for in-class and out-of-class instances, and significantly improve the detection performance in return. Motivated by this intuition, we propose Semi-Supervised Open Set Classification algorithm (S2OSC), a transductive classifier learning process to mitigate embedding confusion. To the best of our knowledge, none of the previous work has addressed this detection manner. At a high-level, S2OSC can also be adapted to incremental OSC conveniently.

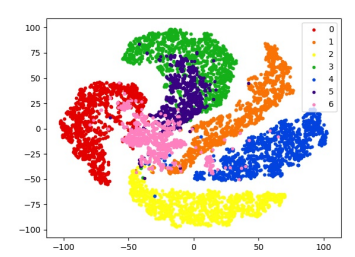

(a) DM on MNIST

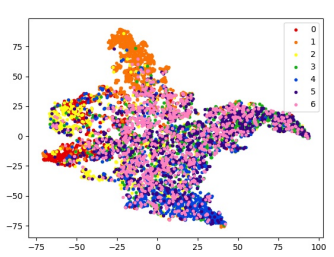

(b) DM on CIFAR-10

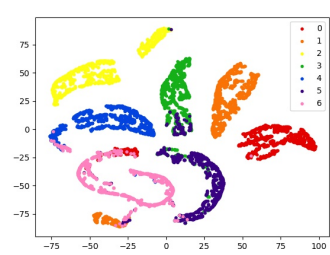

(c) GM on MNIST

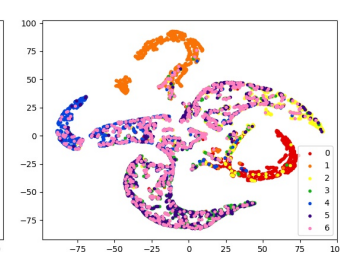

(d) GM on CIFAR-10

Figure 1: T-SNE of discriminative model (DM) [8] and generative model (GM) [11] on simple (MNIST) and complex (CIFAR-10) datasets. We develop these two models with five classes (i.e., $0-4)$ in training stage according to the raw paper, then utilize the pre-trained models to achieve the embeddings of known classes (i.e., 0-4) and unknown classes (i.e., 5 and 6) appearing in testing stage. Note that traditional OSC methods usually classify based on the learned embeddings. 


\section{Related Work}

To set forth the S2OSC, we first introduce existing methods for OSC, which are related to our S2OSC, i.e., discriminative and generative models. Then we present traditional anomaly detection and zero-shot learning methods.

Discriminative OSC models. These approaches mainly restrict intra-class and inter-class distance property on training data, and detect unknown classes by identifying outliers. For example, [12] developed a SVM-based method, which learned the concept of known classes while incorporating the structure presented in unlabeled data from open set; [13] proposed to dynamically maintain two low-dimensional matrix sketches to detect emerging new classes. However, these linear approaches are difficult to process high dimensional space. Recently, several studies have applied deep learning techniques to OSC scenario. For example, [7] distinguished known/unknown class with softmax output probabilities; [14] directly utilized temperature scaling to separate the softmax score between in-distribution and out-of-distribution images; [8] proposed a cnn-based prototype ensemble method, which adaptively updates prototype for robust detection. However, these methods can hardly consider the out-of-class instances in training phase.

Generative OSC models. The key component of generation-based OSC models is to generate effective out-of-class instances. For example, [9] proposed the generative OpenMax (G-OpenMax) algorithm, which provides probability estimation over generated out-of-class instances, that enables the classifier to locate the decision margin according to both in-class and out-of-class knowledge; [10] adopted the GAN technique to generate fake data considering representativeness as the out-ofclass data, which can further enhance the robustness of classifier for detection; [11] introduced an augmentation technique, which adopts an encoder-decoder GAN architecture to generate synthetic instances similar to known classes. Though these methods have achieved some improvements, generating more effective out-of-class instances with complex content still need further research [11].

Traditional detection models. Anomaly detection and generalized zero-shot learning are also related to OSC task. The goal of anomaly detection is to separate outlier instances, for example, [2] proposed a non-parametric method IForest, which detects outliers with ensemble trees. However, anomaly detection follows different protocols from OSC methods, and unable to subdivide known classes. Generalized zero-shot learning aims to classify known and unknown classes with side information. For example, [4] employed manifold learning to align semantic space with visual features; [5] introduced the feature confusion GAN, which adopts a boundary loss to maximize the margin of known and unknown classes. However, they assume that semantic information of unknown classes is already in existence, which is incomparable with OSC methods.

\section{Our Algorithm: S2OSC}

\subsection{Problem Definition}

Without any loss of generality, suppose we have a supervised training set $\mathcal{D}_{t r}=\left\{\left(\mathbf{x}_{i}, \mathbf{y}_{i}\right)\right\}_{i=1}^{N_{t r}}$ at initial time, where $\mathbf{x}_{i} \in \mathcal{R}^{d}$ denotes the $i$-th instance, and $\mathbf{y}_{i} \in Y=\{1,2, \cdots, C\}$ denotes the corresponding label. Then, we receive a pool of unlabeled testing data $\mathcal{D}_{t e}=\left\{\left(\mathbf{x}_{j}\right)\right\}_{j=1}^{N_{t e}}$, where $\mathbf{x}_{j} \in \mathcal{R}^{d}$ denotes the $j-$ th instance, and label $\mathbf{y}_{j} \in \hat{Y}=\{1,2, \cdots, C, C+1, \cdots, C+B\}$ is unknown. $\{1,2, \cdots, C\}$ denotes in-class set and $\{C+1, \cdots, C+B\}$ represents out-of-class set. Therefore, open set classification can be defined as:

Definition 1 Open Set Classification (OSC) With the initial training set $\mathcal{D}_{t r}=\left\{\left(\mathbf{x}_{i}, \mathbf{y}_{i}\right)\right\}_{i=1}^{N_{t r}}$, we aim to construct a model $f: X \rightarrow Y$. Then with the pre-trained model $f$, OSC is to classify the in-class and out-of-class instances in $\mathcal{D}_{\text {te }}$ accurately.

Following most OSC approaches [12, 13,11,14, 8, 1], we firstly consider all unknown classes as a super-class for detection, then employ unsupervised clustering techniques such as k-means for subdividing (out-of-class specifically refers to super-class in following). Therefore, given the $f$ and $\mathcal{D}_{t e}$, we transform to build a new classifier $g$ in transductive manner for operating OSC on $\mathcal{D}_{t e}$. In detail, S2OSC pre-trains a classification model $f$ with $\mathcal{D}_{t r}$ and stores limited in-class examples $\mathcal{D}_{i n}$ from $\mathcal{D}_{t r} . f$ is then used for filtering distinct out-of-class instances $\mathcal{D}_{\text {out }}$ in $\mathcal{D}_{\text {te }}$. After this, we possess in-class and potential out-of-class labeled data $\mathcal{X}=\left\{\mathcal{D}_{\text {in }}, \mathcal{D}_{\text {out }}\right\}$, and unlabeled data 
$\mathcal{U}=\left\{\mathcal{D}_{\text {te }} \backslash \mathcal{D}_{\text {out }}\right\}$, thereby we can develop a new classifier $g$ in semi-supervised paradigm. Note that there are two ways to train $g$ : 1) fine-tuning based on $f$ directly; 2) retraining from scratch while using $f$ as a teacher for knowledge distillation. We select the second way considering the efficiency and effectiveness. Consequently, we acquire the classification results of $\mathcal{D}_{t e}$ using learned $g$ in a transductive manner. In fact, S2OSC comprehensively considers the ideas of both discriminant and generative methods, i.e., trying to separate known classes as far as possible, while taking into account the potential information of unknown classes. Next, we describe each part of S2OSC.

\subsection{Data Filtering}

With the initial in-class training data $\mathcal{D}_{t r}$, we firstly develop a deep classification model $f$ as many typical supervised methods:

$$
f^{*}=\arg \min _{f} \sum_{i=1}^{N_{t r}} \ell\left(\mathbf{y}_{i}, f\left(\mathbf{x}_{i}\right)\right)
$$

$\ell$ can be any convex loss function, and we define as cross-entropy loss for unification here. Meanwhile, we randomly select $K$ examples from each class to constitute $\mathcal{D}_{i n}$. Then, we evaluate the weight of each instance in $\mathcal{D}_{t e}$ by self-taught weighting function. In detail, we compute confidence score for each instance $\mathbf{x}_{j}$ in $\mathcal{D}_{\text {te }}$ using pre-trained model $f^{*}$ :

$$
w_{j}=u_{j}+\lambda d_{j}
$$

where $\lambda$ is a fixed hyperparameter. $u_{j}$ denotes statistic prediction confidence, which is done explicitly with the entropy: $u_{j}=-\sum_{c} f_{c}^{*}\left(\mathbf{x}_{j}\right) \log f_{c}^{*}\left(\mathbf{x}_{j}\right) . d_{j}$ represents statistic distance to each in-class center, i.e., $d_{j}=\min \left(\left\|e_{j}-\mu_{c}\right\|_{2}^{2}\right)$, where $e_{j}$ represents embeddings extracted from feature output layer of $f^{*}$, and $\mu_{c}=\frac{1}{\left|\mathcal{D}_{t r}^{c}\right|} \sum_{\mathbf{x} \in \mathcal{D}_{t r}^{c}} e_{\mathbf{x}}$ represents $c$-th in-class center, $\mathcal{D}_{t r}^{c}$ is $c$-th class set. It is notable that highly unsure out-of-class instances have larger weights, while in-class and confused instances have lower weights. In result, we can sort $\mathcal{D}_{t e}$ according to $w$, and acquire filtered instances set $\mathcal{D}_{\text {out }}$ with the same number $K$ as in-class set, the corresponding super-class is $C^{\prime}$. Therefore, we have owned in-class and out-of-class labeled data $\mathcal{X}=\left\{\mathcal{D}_{\text {in }}, \mathcal{D}_{\text {out }}\right\}$, unlabeled data $\mathcal{U}=\left\{\mathcal{D}_{\text {te }} \backslash \mathcal{D}_{\text {out }}\right\}$, and aim to develop the new classifier $g$.

\subsection{Objective Function}

Inspired from [15], we combine two common semi-supervised methods to learn $g$ : consistency regularization and pseudo-labeling, which aim to effectively utilize unlabeled data by ensuring the consistency among different data-augmented forms. S2OSC has two contributions: 1) Pseudolabeling threshold. For a given unlabeled instance, the pseudo-label is only retained if $g$ produces a high-confidence prediction; 2) Pre-trained model teaching. For a given instance, we use the pretrained model $f$ for knowledge distillation of predictions from known classes. Therefore, we can further separate the confused out-of-class instances with in-class instances.

Specifically, the loss function of $g$ exclusively consists of two terms: a supervised loss $L_{s}$ applied to labeled data and an unsupervised loss $L_{u} . L_{s}$ can be represented as:

$$
\begin{aligned}
L_{s} & =\frac{1}{2|\mathcal{X}|} \sum_{l=1}^{|\mathcal{X}|}\left(\ell_{s 1}\left(\mathbf{x}_{l}, \mathbf{y}_{l}\right)+\alpha \ell_{s 2}\left(\mathbf{x}_{l}, f^{*}\left(\mathbf{x}_{l}\right)\right)\right) \\
\ell_{s 1} & =H_{\text {in }}\left(\mathbf{y}_{l}, g\left(\mathbf{x}_{l}\right)\right)+\mathbf{1}_{\max \left(g\left(\mathbf{x}_{l}\right)\right) \geq \tau} H_{\text {out }}\left(\mathbf{y}_{l}, g\left(\mathbf{x}_{l}\right)\right) \\
\ell_{s 2} & =K L\left(f^{*}\left(\mathbf{x}_{l}\right) \| g_{\backslash C^{\prime}}\left(\mathbf{x}_{l}\right)\right)
\end{aligned}
$$

where $H_{(\cdot)}(p, q)=-p \log q$ is standard cross-entropy loss, $K L(p \| q)=p \log \frac{p}{q}$ denotes KLdivergence. $\alpha$ is a hyperparameter, $\tau$ is a scalar parameter denoting the threshold. $g_{\backslash C^{\prime}}\left(\mathbf{x}_{l}\right)$ is the prediction distribution with re-softmax except out-of-class $C^{\prime} . \ell_{s 1}$ adopts the standard crossentropy loss, note that there may still have embedding confused known class data in $\mathcal{D}_{\text {out }}$, thus we utilize $\mathbf{1}_{\max \left(g\left(\mathbf{x}_{l}\right)\right) \geq \tau}$ term to produce a valid "one-hot" probability distribution. Meanwhile, ideally, for labeled known class data in $\mathcal{X}, f$ can also produce confident probability distribution, otherwise $f$ tends to predict uniform distribution. Thus, $\ell_{s 2}$ receives the soft targets from $f$ for 
in-class and out-of-class examples, which aim to proceed knowledge distillation by restraining two prediction distribution. $f^{*}\left(\mathbf{x}_{l}\right)$ and $g \backslash C^{\prime}\left(\mathbf{x}_{l}\right)$ is with Softmax-T that sharpens distribution by adjusting its temperature $T$ following [16], i.e., raising all probabilities to a power of $\frac{1}{T}$ and re-normalizing.

For unlabeled data, S2OSC firstly obtains the pseudo-label by computing the prediction for a given unlabeled instance: $q_{u}=g\left(\mathbf{x}_{u}\right)$, and $\hat{q}_{u}=\arg \max \left(q_{u}\right)$ is the pseudo-label, which is then used to enforce the loss against model's output for a augmented version of $\mathbf{x}_{u}$ :

$$
\begin{aligned}
L_{u} & =\frac{1}{2|\mathcal{U}|} \sum_{u=1}^{|\mathcal{U}|} \mathbf{1}_{\max \left(q_{u}\right) \geq \tau}\left(\ell_{u 1}\left(\mathbf{x}_{u}, \hat{q}_{u}\right)+\alpha \ell_{u 2}\left(\mathbf{x}_{u}, f^{*}\left(\mathbf{x}_{u}\right)\right)\right) \\
\ell_{u 1} & =H\left(\hat{q}_{u}, g\left(\Phi\left(\mathbf{x}_{u}\right)\right)\right) \\
\ell_{u 2} & =K L\left(f^{*}\left(\mathbf{x}_{u}\right) \| g_{\backslash C^{\prime}}\left(\Phi\left(\mathbf{x}_{u}\right)\right)\right)
\end{aligned}
$$

where $\tau$ denotes threshold above which we retrain the pseudo-label. $\Phi$ represents weak augmentation using a standard flip-and-shift strategy or strong augmentation using CTAugment [17] with Cutout [18] as [15], we employ the former strategy considering efficiency. See Appendix for details. $\ell_{u 2}$ employs similar function on unlabeled data. Thus, $L_{u}$ encourage the model's predictions to be low-entropy (i.e., high-confidence) on unlabeled data combing hard-label and soft-label. In summary, the loss minimized by S2OSC is: $L=L_{s}+\lambda_{u} L_{u}$, where $\lambda_{u}$ is a fixed scalar hyperparameter denoting the relative weight. Consequently, the trained $g$ can classify in-class or out-of-class instances in $D_{t e}$, and then employ clustering on out-of-class instances to acquire sub-classes.

\section{Incremental S2OSC (I-S2OSC)}

In this section, we aim to demonstrate that S2OSC can be extended into incremental open set classification scenario conveniently.

\subsection{Problem Definition}

In real applications, we always receive the streaming data, and unknown classes are also emerge incrementally. Thus more generalized setting is incremental OSC, which has two characteristics: 1) Data pool. At time window $t$, we only get the data of current time window, i.e., $\mathcal{D}_{t e}^{t}$, not full amount of previous data; 2) Unknown class continuity. At time window $t$, unknown classes appear partially, thereby we need to incrementally conduct OSC, i.e., every time after receiving the data of time window $t$, OSC is performed. Specifically, the streaming data $\mathcal{D}$ can be divided into $\mathcal{D}=\left\{\mathcal{D}^{t}\right\}_{t=0}^{T}$, where $\mathcal{D}^{0}=\mathcal{D}_{t r}$ is the initial training set. $\mathcal{D}^{t}=\left\{\mathbf{x}_{j}^{t}\right\}_{j=1}^{N_{t}}, t \geq 1$ is with $N_{t}$ unlabeled instances, and the underlying label $\mathbf{y}_{j}^{t} \in \hat{Y}^{t}$ is unknown, $\hat{Y}^{t}=\hat{Y}^{t-1} \cup Y^{t}$, where $\hat{Y}^{t-1}$ is the cumulative known classes until $(t-1)$-th time window and $Y^{t}$ is the unknown class set in $t$-th window. Therefore, we provide the definition of incremental open set classification:

Definition 2 Incremental Open Set Classification (IOSC) At time $t \in\{1,2, \cdots, T\}$, we have pretrained model $f^{t-1}$ and limited stored in-class examples $M^{t-1}$ until $(t-1)$-th time, then receive newly coming data pool $\mathcal{D}^{t}$. First, we aim to classify known and unknown classes in $\mathcal{D}^{t}$ as Definition 1. Then, with the labeled data from novel classes and stored data $M^{t-1}$, we update the model while mitigating forgetting to acquire $f^{t}$. Cycle this process until terminated.

S2OSC can be applied directly for OSC of $\mathcal{D}^{t}$ at $t$-th time window, then the extra challenge is to update the model while mitigating forgetting [19] of previous in-class knowledge.

\subsection{Model Update}

There exist two labeling cases after OSC, i.e., manually labeling and self-taught labeling [13]. We consider first setting following most approaches $[1,13,8]$ to avoid label noise accumulation. In detail, after known/unknown classification operator, we can achieve potential out-of-class instances to query their true labels. However, there exist catastrophic forgetting of known classes if we only use the new data to update the model. To solve this problem, we employ a mechanism to incorporate the stored memory and novel class information incrementally, which can mitigate forgetting of 
Table 1: Comparison of open set classification performance.

\begin{tabular}{|c|c|c|c|c|c|c|c|c|c|c|}
\hline \multirow{2}{*}{ Methods } & \multicolumn{5}{|c|}{ Accuracy } & \multicolumn{5}{|c|}{ F1 } \\
\hline & F-MN & CIFAR & SVHN & $\mathrm{MN}$ & CINIC & F-MN & CIFAR & SVHN & $\mathrm{MN}$ & CINIC \\
\hline Iforest & .554 & .243 & .198 & .632 & .240 & .553 & .243 & .197 & .625 & .240 \\
\hline One-SVM & .474 & .260 & .195 & .537 & .243 & .671 & .223 & .102 & .520 & .206 \\
\hline LACU & .394 & .325 & .193 & .695 & .303 & .409 & .326 & .091 & .681 & .268 \\
\hline SENC & .420 & .215 & .184 & .358 & .230 & .489 & .171 & .124 & .302 & .166 \\
\hline ODIN & .563 & .426 & .601 & .778 & .329 & .854 & .380 & .584 & .767 & .243 \\
\hline $\mathrm{CFO}$ & .639 & .502 & .663 & .514 & .389 & .720 & .514 & .656 & .513 & .359 \\
\hline $\mathrm{CPE}$ & .628 & .438 & .645 & .961 & .246 & .605 & .353 & .791 & .960 & .270 \\
\hline DTC & .576 & .363 & .534 & .741 & .388 & .665 & .495 & .606 & .717 & .452 \\
\hline S2OSC & .972 & .847 & .898 & .985 & .785 & .972 & .854 & .901 & .985 & .787 \\
\hline \multirow{2}{*}{ Methods } & \multicolumn{5}{|c|}{ Precision } & \multicolumn{5}{|c|}{ Recall } \\
\hline & F-MN & CIFAR & SVHN & $\mathrm{MN}$ & CINIC & F-MN & CIFAR & SVHN & $\mathrm{MN}$ & CINIC \\
\hline Iforest & .553 & .554 & .252 & .243 & .198 & .197 & .657 & .632 & .245 & .240 \\
\hline One-SVM & .671 & .474 & .286 & .260 & .195 & .102 & .616 & .537 & .274 & .243 \\
\hline LACU & .409 & .394 & .331 & .325 & .193 & .091 & .676 & .695 & .363 & .303 \\
\hline SENC & .489 & .420 & .253 & .215 & .184 & .124 & .448 & .358 & .211 & .230 \\
\hline$\overline{\text { ODIN }}$ & .854 & .563 & .520 & .426 & .601 & .584 & .878 & .778 & .554 & .329 \\
\hline $\mathrm{CFO}$ & .720 & .639 & .579 & .502 & .663 & .656 & .598 & .514 & .436 & .389 \\
\hline CPE & .605 & .698 & .336 & .408 & .645 & .791 & .955 & .961 & .302 & .316 \\
\hline DTC & .665 & .576 & .435 & .463 & .434 & .606 & .699 & .681 & .428 & .388 \\
\hline S2OSC & .972 & .972 & .888 & .847 & .898 & .901 & .986 & .985 & .799 & .785 \\
\hline
\end{tabular}

discriminatory characteristics about known classes. Specifically, we utilize the exemplary data $M^{t-1}$ for regularization in fine-tuning:

$$
\begin{aligned}
& L^{t}=\sum_{l} \ell\left(\mathbf{y}_{l}, f^{t}\left(\mathbf{x}_{l}\right)\right) \\
& \text { s.t. } \quad \ell\left(M^{t-1}, f^{t}\right) \leq \ell\left(M^{t-1}, f^{t-1}\right)
\end{aligned}
$$

The loss term encourages the labeled unknown class examples to fine-tune $f^{t-1}$ for better performance, while constraint term imposes $M^{t-1}$ for less forgetting of old in-class knowledge. We utilize directly joint optimization on $M^{t-1}$ as iCaRL [20] to optimize variant of Eq. 5. See Appendix for details. After that, we need to update the $M^{t-1}$ to store key points of unknown classes. If $M^{t-1}$ is not full, we can fill selected instances from unknown class directly. Otherwise, we remove equal instances for each known class, and fill instances for each unknown class.

\section{Experiments}

\subsection{Datasets and Baselines}

Considering that incremental OSC is an extension of OSC, the incremental OSC methods can also be applied to the setting of OSC. Therefore, we adopt commonly used incremental OSC datasets for validation here. In detail, we utilize five visual datasets in this paper following [8], i.e., FASHIONMNIST (F-MN) [21], CIFAR-10 (CIFAR) [22], SVHN [23], MNIST (MN) [24], CINIC ${ }^{2}$. To validate the effectiveness of proposed approach, we compared it with existing state-of-the-art OSC and incremental OSC methods. First, we compare it with traditional anomaly detection and linear methods: Iforest [2], One-Class SVM (One-SVM) [25], LACU-SVM (LACU) [12], SENC-MAS (SENC) [13]. Second, we compare it with recent deep methods: ODIN-CNN (ODIN) [14], CFO [11], CPE [8] and DTC [27]. Abbreviations in parentheses. DTC is clustering based methods for multiple unknown classes detection. Note that Iforest, One-SVM, LACU, ODIN, CFO, and DTC are OSC

\footnotetext{
${ }^{2}$ https://github.com/BayesWatch/cinic-10
} 
methods, SENC and CPE are incremental OSC methods. All OSC baselines except Iforest can be updated incrementally using newly labeled unknown class data and memory data.

\subsection{Open Set Classification}

To rearrange each dataset for emulating a OSC form, we randomly hold out $50 \%$ classes as initial training set, and leave one class for testing. Experiments about OSC with various number of outof-classes can refer to supplementary materials. Moreover, we extracted 33\% of the known class data into test set, so that the test set mixes with known and unknown classes. Here we utilize four commonly used criteria, i.e., Accuracy, Precision, Recall and F1 (Weighted F1), to measure the classification performance, which considers all known and unknown classes. For example, accuracy $A=\frac{\sum_{i=1}^{|\hat{Y}|}\left(T P_{i}+T N_{i}\right)}{\sum_{i=1}^{\mid \hat{Y}} T P_{i}+T N_{i}+F P_{i}+F N_{i}}$, where $T P, F P, F N, T N$ denotes the true positives, false positive, false negatives and true negatives.

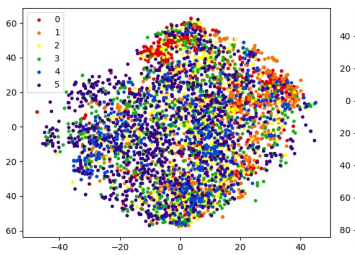

(a) Original

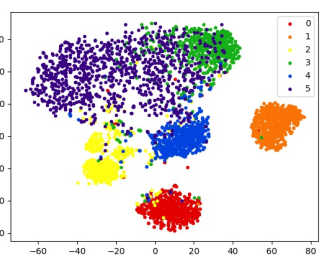

(b) S2OSC

Figure 2: T-SNE Visualization on CIFAR-10 dataset. (a) original feature space; (b) learned embeddings by proposed S2OSC.

improvements than baselines. This indicates the effectivenets mitigating embedding confusion. Figure 2 shows feature embedding results using T-SNE with the similar setting in Figure 1. Clearly, the figure (b) shows that the output of S2OSC has learned distinct groups, which is much better than original embeddings and corresponding embeddings of other deep methods in Figure 1. This validates that instances from unknown classes are well separated from other known clusters, which benefits for unknown class detection.

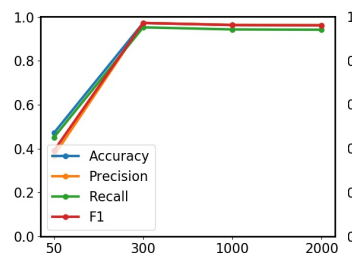

(a) Fashion-MNIST

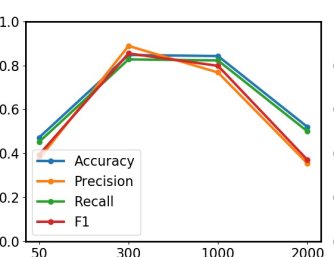

(b) CIFAR-10

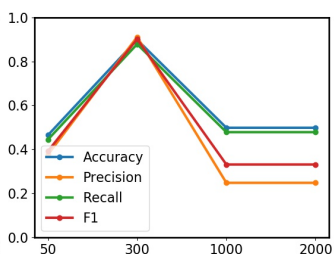

(c) $\mathrm{SVNH}$

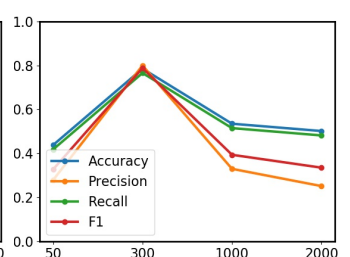

(d) CINIC

Figure 3: Classification performance with various number of filtered out-of-class instances.

Figure 3 shows the influence of important parameter $K$ (filtering size), i.e., we tune the size of $K=\{50,300,1000,2000\}$. The results reveal that, at first, different criteria improve with the increase of filtered instances. After filtering size is more than a threshold, the performance start to decrease. For example, on CIFAR-10, the accuracy on 300 filtering is about $84.7 \%$, yet the performance decreases after 300 filtering, this is due to the introduction of embedding confused instances with the increase of $K$.

\subsection{Incremental Open Set Classification}

Furthermore, we rearrange instances in each dataset to emulate a streaming form with incremental unknown classes as [8]. We utilize the same four criteria, i.e., average Accuracy, average Precision, average Recall and average F1, over various data pools to measure the performance following [8], 
Table 2: Comparison of incremental open set classification performance.

\begin{tabular}{|c|c|c|c|c|c|c|c|c|c|c|}
\hline \multirow{2}{*}{ Methods } & \multicolumn{5}{|c|}{ Average Accuracy } & \multicolumn{5}{|c|}{ Average F1 } \\
\hline & F-MN & CIFAR & SVHN & $\mathrm{MN}$ & CINIC & F-MN & CIFAR & SVHN & $\mathrm{MN}$ & CINIC \\
\hline Iforest & .533 & .221 & .170 & .606 & .220 & .489 & .217 & .162 & .595 & .217 \\
\hline One-SVM & .404 & .218 & .167 & .471 & .205 & .409 & .169 & .085 & .476 & .156 \\
\hline LACU & .332 & .199 & .149 & .171 & .188 & .267 & .137 & .052 & .088 & .114 \\
\hline SENC & .341 & .197 & .156 & .296 & .197 & .264 & .145 & .100 & .251 & .138 \\
\hline ODIN & .780 & .334 & .625 & .853 & .276 & .767 & .284 & .593 & .850 & .206 \\
\hline CFO & .659 & .306 & .485 & .745 & 291 & .635 & .304 & .472 & .722 & .283 \\
\hline CPE & .618 & .368 & .695 & .961 & .286 & .599 & .343 & .701 & .960 & .260 \\
\hline DTC & .586 & .393 & .514 & .711 & .348 & .609 & .445 & .566 & .717 & .382 \\
\hline I-S2OSC & .818 & .660 & .771 & .926 & .567 & .774 & .609 & .732 & .913 & .517 \\
\hline \multirow{2}{*}{ Methods } & \multicolumn{5}{|c|}{ Average Precision } & \multicolumn{5}{|c|}{ Average Recall } \\
\hline & F-MN & CIFAR & SVHN & $\mathrm{MN}$ & CINIC & F-MN & CIFAR & SVHN & $\mathrm{MN}$ & CINIC \\
\hline Iforest & .498 & .503 & .226 & .221 & .166 & .170 & .621 & .607 & .225 & .221 \\
\hline One-SVM & .597 & .405 & .216 & .219 & .187 & .167 & .672 & .472 & .210 & .205 \\
\hline LACU & .262 & .332 & .133 & .200 & .043 & .150 & .266 & .172 & .143 & .188 \\
\hline SENC & .330 & .342 & .207 & .198 & .210 & .157 & .390 & .297 & .197 & .198 \\
\hline ODIN & .858 & .781 & .456 & .334 & .672 & .626 & .920 & .853 & .372 & .276 \\
\hline $\mathrm{CFO}$ & .634 & .659 & .307 & .306 & .477 & .486 & .803 & .745 & .285 & .291 \\
\hline CPE & .666 & .619 & .427 & .368 & .762 & .696 & .965 & .961 & .332 & .286 \\
\hline DTC & .666 & .586 & .536 & .394 & .657 & .515 & .800 & .711 & .469 & .349 \\
\hline I-S2OSC & .759 & .818 & .597 & .661 & .718 & .771 & .893 & .922 & .509 & .568 \\
\hline
\end{tabular}

Table 3: Forgetting measure of known classes over streaming data.

\begin{tabular}{l|c|c|c|c|c|c|c|c|c}
\hline \multirow{2}{*}{ Methods } & \multicolumn{10}{c}{ Forgetting } \\
\cline { 2 - 9 } & Iforest & One-SVM & LACU & SENC & ODIN & CFO & CPE & DTC & I-S2OSC \\
\hline F-MN & N/A & .139 & .121 & .127 & .107 & .047 & .032 & .055 & $\mathbf{. 0 2 9}$ \\
CIFAR & N/A & .202 & .127 & .172 & .132 & .128 & .118 & .120 & $\mathbf{. 1 1 7}$ \\
SVHN & N/A & .243 & .330 & .249 & .168 & .130 & .124 & .159 & $\mathbf{. 1 2 3}$ \\
MN & N/A & .141 & .080 & .061 & .049 & .040 & $\mathbf{. 0 3 3}$ & .044 & .037 \\
CINIC & N/A & .224 & .227 & .278 & .170 & .190 & .147 & .175 & $\mathbf{. 1 3 8}$ \\
\hline
\end{tabular}

which aims to calculate the overall performance for streaming data. Moreover, to validate the catastrophic forgetting of $f$, we calculate the performance about forgetting profile of different learning algorithms as [28], which defines the difference between maximum knowledge gained of emerging classes on a particular window throughout the learning process and we currently have about it, the lower difference the better.

Table 2 compares the classification performance of I-S2OSC with all baselines on streaming data. Table 3 compares the forgetting performance. "N/A" denotes no results considering that Iforest has no update process. We observe that: 1) comparing with results in Table 1, most average classification metrics of deep methods have improved while linear methods decreased, this indicates that deep models can still effectively distinguish known classes for streaming data, which can further benefit OSC; 2) I-S2OSC is superior than other baselines over accuracy and F1 metrics except MNIST dataset, and other two metrics are competitive. But it is not as obvious as the effect in OSC setting. Besides, the average performance of I-S2OSC decreases comparing OSC setting. These phenomenons are because that we uniformly set $K$ to 300, and with the increase of emerging classes, the number of inclusive in-class in filtering data also increases, which will affect the training of $g$. Thus the value of $K$ needs to be tuned carefully; 3) I-S2OSC has the smallest forgetting except MMIST dataset by considering exemplary regularization, which benefits to preserve known class knowledge. 


\section{Conclusion}

Real-word applications always receive the data with unknown classes, thus it is necessary to promote the open set classification. The key challenge in OSC is to overcome the embedding confusion caused by out-of-class instances. To this end, we propose a holistic semi-supervised OSC algorithm, S2OSC. S2OSC incorporated out-of-class instances filtering and semi-supervised model training in a transductive manner, and integrated in-class pre-trained model for teaching. Moreover, S2OSC can be adapted to incremental OSC setting efficiently. Experiments showed the superior performances of S2OSC and I-S2OSC.

\section{References}

[1] Geng, C., S. Huang, S. Chen. Recent advances in open set recognition: A survey. CoRR, abs/1811.08581, 2018.

[2] Liu, F. T., K. M. Ting, Z. Zhou. Isolation forest. In ICDM, pages 413-422. 2008.

[3] Xia, Y., X. Cao, F. Wen, et al. Learning discriminative reconstructions for unsupervised outlier removal. In ICCV, pages 1511-1519. 2015.

[4] Changpinyo, S., W.-L. Chao, B. Gong, et al. Synthesized classifiers for zero-shot learning. In CVPR, pages 5327-5336. 2016.

[5] Li, J., M. Jing, K. Lu, et al. Alleviating feature confusion for generative zero-shot learning. In ACMMM, pages 1587-1595. 2019.

[6] Cai, X., P. Zhao, K. Ting, et al. Nearest neighbor ensembles: An effective method for difficult problems in streaming classification with emerging new classes. In ICDM, pages 970-975. 2019.

[7] Hendrycks, D., K. Gimpel. A baseline for detecting misclassified and out-of-distribution examples in neural networks. In ICLR. 2017.

[8] Wang, Z., Z. Kong, S. Chandra, et al. Robust high dimensional stream classification with novel class detection. In ICDE, pages 1418-1429. 2019.

[9] Ge, Z., S. Demyanov, R. Garnavi. Generative openmax for multi-class open set classification. In $B M V C$. 2017.

[10] Jo, I., J. Kim, H. Kang, et al. Open set recognition by regularising classifier with fake data generated by generative adversarial networks. In ICASSP, pages 2686-2690. 2018.

[11] Neal, L., M. L. Olson, X. Z. Fern, et al. Open set learning with counterfactual images. In ECCV, pages 620-635. 2018.

[12] Da, Q., Y. Yu, Z.-H. Zhou. Learning with augmented class by exploiting unlabeled data. In AAAI, pages 1760-1766. 2014.

[13] Mu, X., F. Zhu, J. Du, et al. Streaming classification with emerging new class by class matrix sketching. In AAAI, pages 2373-2379. 2017.

[14] Liang, S., Y. Li, R. Srikant. Enhancing the reliability of out-of-distribution image detection in neural networks. In ICLR. 2018.

[15] Sohn, K., D. Berthelot, C. Li, et al. Fixmatch: Simplifying semi-supervised learning with consistency and confidence. CoRR, abs/2001.07685, 2020.

[16] Hinton, G. E., O. Vinyals, J. Dean. Distilling the knowledge in a neural network. CoRR, abs/1503.02531, 2015.

[17] Berthelot, D., N. Carlini, E. D. Cubuk, et al. Remixmatch: Semi-supervised learning with distribution matching and augmentation anchoring. In ICLR. 2020.

[18] Devries, T., G. W. Taylor. Improved regularization of convolutional neural networks with cutout. CoRR, abs/1708.04552, 2017.

[19] Ratcliff, R. Connectionist models of recognition memory: constraints imposed by learning and forgetting functions. Psychol. Review, 97(2):285, 1990. 
[20] Rebuffi, S.-A., A. Kolesnikov, G. Sperl, et al. icarl: Incremental classifier and representation learning. In CVPR, pages 5533-5542. 2017.

[21] Xiao, H., K. Rasul, R. Vollgraf. Fashion-mnist: a novel image dataset for benchmarking machine learning algorithms. CoRR, abs/1708.07747, 2017.

[22] Krizhevsky, A., G. Hinton, et al. Learning multiple layers of features from tiny images. 2009.

[23] Netzer, Y., T. Wang, A. Coates, et al. Reading digits in natural images with unsupervised feature learning. NeurIPS Workshop, 2011(2):5, 2011.

[24] LeCun, Y., C. Cortes, C. J. Burges. The mnist database of handwritten digits, 1998. URL http://yann. lecun. com/exdb/mnist, 10:34, 1998.

[25] Scholkopf, B., J. C. Platt, J. Shawe-Taylor, et al. Estimating the support of a high-dimensional distribution. Neural Computation, 13(7):1443-1471, 2001.

[26] Hsu, Y., Z. Lv, J. Schlosser, et al. Multi-class classification without multi-class labels. In ICLR. 2019.

[27] Han, K., A. Vedaldi, A. Zisserman. Learning to discover novel visual categories via deep transfer clustering. In $I C C V$, pages 8400-8408. 2019.

[28] Chaudhry, A., P. K. Dokania, T. Ajanthan, et al. Riemannian walk for incremental learning: Understanding forgetting and intransigence. CoRR, abs/1801.10112, 2018. 


\title{
Supplementary Material of S2OSC: A Holistic Semi-Supervised Approach for Open Set Classification
}

\author{
Anonymous Author(s) \\ Affiliation \\ Address \\ email
}

\section{The Algorithm Pipeline}

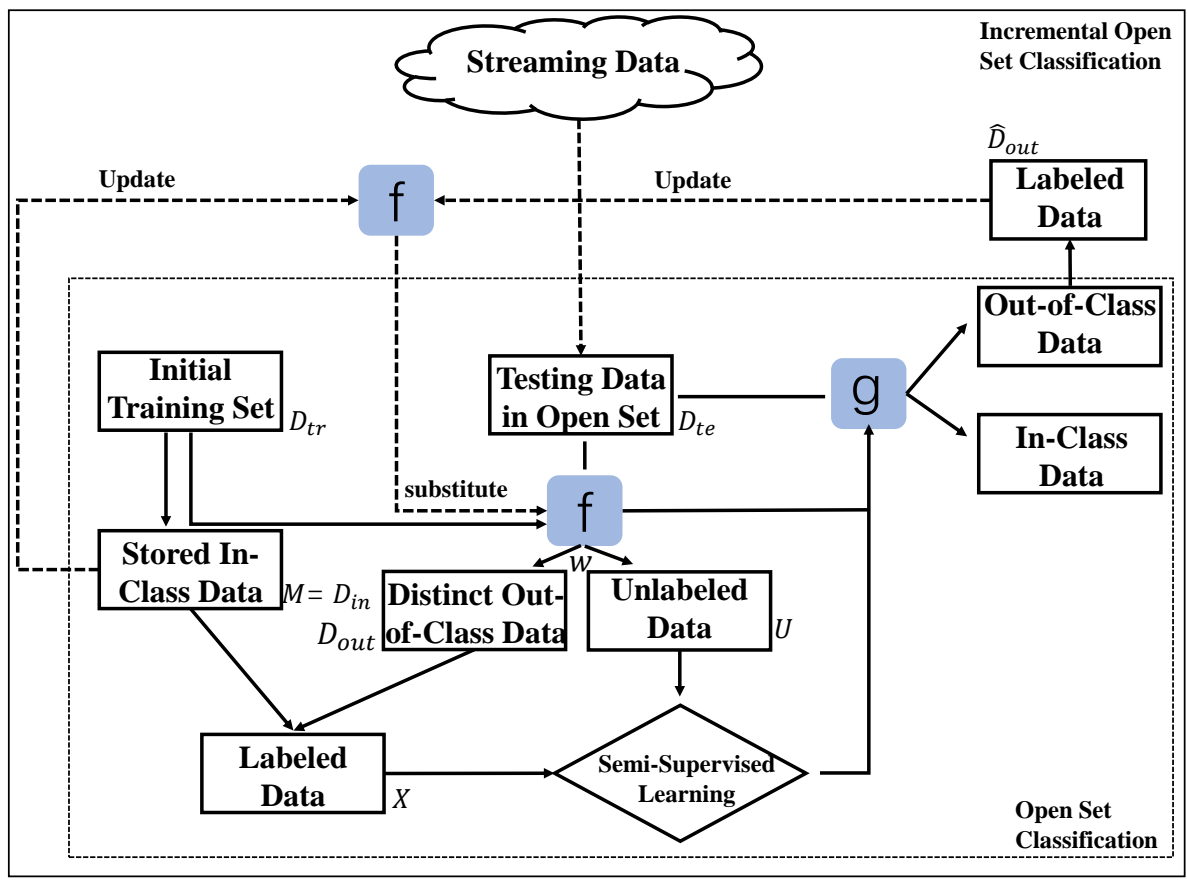

Figure 1: Pipeline of the S2OSC and I-S2OSC.

2 In this section, we provide the pipeline of S2OSC and I-S2OSC as shown in Figure 1, where the dotted frame part is the S2OSC pipeline, and the solid line part is the extended I-S2OSC. Specifically, look from top to bottom inside the dotted frame. Given a set of initially in-class training set $\mathcal{D}_{t r}$, we carry on with two jobs: 1$)$ pre-train an in-class classification model $f$; 2) store limited in-class examples $\mathcal{D}_{i n}$. Then, we receive the testing data $\mathcal{D}_{t e}$ from open set, and $\mathcal{D}_{t e}$ can be divided into two parts using $f: 1)$ distinct out-of-class instances $\left.\mathcal{D}_{\text {out }} ; 2\right)$ unlabeled data $\mathcal{U}=\left\{\mathcal{D}_{\text {te }} \backslash \mathcal{D}_{\text {out }}\right\}$. Here all instances in $\mathcal{D}_{\text {out }}$ are reduced to an unified super-class. In result, we possess in-class and out-of-class labeled data $\mathcal{X}=\left\{\mathcal{D}_{\text {in }}, \mathcal{D}_{\text {out }}\right\}$ and unlabeled data $\mathcal{U}=\left\{\mathcal{D}_{\text {te }} \backslash \mathcal{D}_{\text {out }}\right\}$, thereby we can develop a new classifier $g$ in semi-supervised paradigm by considering $f$ as teacher model simultaneously. And we can acquire the classification results of $\mathcal{D}_{t e}$ using learned $g$ in a transductive manner. Derived to incremental OSC scenario, we receive the testing data $D^{t}$ of $t$-th time window from the streaming 
data, and utilize S2OSC for open set classification. After that, we query the ground-truths of potential unknown class data, and combine stored in-class data to incrementally update $f^{t}$, then substitute last $f^{t-1}$. It is notable that $g$ is re-trained from scratch for every time window.

\section{Our Algorithm: S2OSC}

\subsection{Weak and Strong Data-augmentation}

In this paper, we leverage the weak and strong augmentation, i.e., $\Phi(\mathbf{x})$, following [1]:

- Weak augmentation. It adopts a standard flip-and-shift strategy. On all datasets, Images is randomly flipped horizontally with a probability of $50 \%$, and randomly translated by up to $12.5 \%$ vertically and horizontally;

- Strong augmentation. It adopts CTAugment [2] with Cutout technique based on AutoAugment [3]. Here, AutoAugment learns an augmentation strategy with reinforcement learning technique, which refers to transformations from the Python Imaging Library ${ }^{1}$, and requires labeled data. CTAugment is a variant of AutoAugment, which requires no labeled data. Further details on CTAugment can be found in [2]. Cutout is a simple regularization technique that randomly masks out square regions of input image.

Therefore, weak augmentation produces slightly distorted version of a given image, while strong augmentation produces heavily distorted version of a given image.

\subsection{S2OSC Considering Multiple Unknown Classes}

As a matter of fact, multiple unknown classes emerging simultaneously have a certain effect on training $g$, that is the pseudo super-class may contains sub-classes with different semantics. Using CIFAR-10 as an example, truck and dog may coexist in unknown class set, and it will lead to separated intra-class distances of unknown super-class. Thus known classes that are semantically similar to unknown classes will affect learning process. To solve this problem, we also turn to unify the known classes into a super-class, which aims to learn the distribution difference between known and unknown class sets. Thus the training of $g$ is transformed into a binary classification problem. In detail, we consider the known class set $\{1,2,3, \cdots, C\}$ as positive class, and unknown class set as negative class. Thus the loss function can be reformulated as:

$$
\begin{aligned}
L & =L_{s}+\lambda_{u} L_{u} \\
L_{s} & =\frac{1}{2|\mathcal{X}|} \sum_{l=1}^{|\mathcal{X}|}\left\{H\left(\hat{\mathbf{y}}_{l}, g\left(\mathbf{x}_{l}\right)\right)+\alpha K L\left(\psi\left(\mathbf{x}_{l}\right), g\left(\Phi\left(\mathbf{x}_{l}\right)\right)\right)\right\} \\
L_{u} & =\frac{1}{2|\mathcal{U}|} \sum_{u=1}^{|\mathcal{U}|} \mathbf{1}_{\max \left(q_{u}\right) \geq \tau}\left\{H\left(\hat{q}_{u}, g\left(\Phi\left(\mathbf{x}_{u}\right)\right)\right)+\alpha K L\left(\psi\left(\mathbf{x}_{l}\right), g\left(\Phi\left(\mathbf{x}_{u}\right)\right)\right)\right\}
\end{aligned}
$$

where $\hat{\mathbf{y}}_{l} \in\{1,0\}$ denotes the re-defined super-class. $H(\cdot)$ denotes the cross-entropy for binary classification. $\psi\left(\mathbf{x}_{l}\right)=\left[1-\ell\left(f^{*}\left(\mathbf{x}_{l}\right)\right), \ell\left(f^{*}\left(\mathbf{x}_{l}\right)\right)\right], \ell\left(f^{*}\left(\mathbf{x}_{l}\right)\right)$ denotes the loss function of $\mathbf{x}$ using $f^{*}, \ell(\cdot)$ is small for known class data, while becomes large for unknown class data. Considering the data balance, a total of $K$ examples are sampled from known classes. With the elementary prediction of learned $g$, we can utilize $f^{*}$ for further sub-dividing of known classes. In experiment, we also combine labeled data into unlabeled dataset. It is notable that we adopt this S2OSC variant to conduct multi-class OSC experiments, and get superior results.

\section{Incremental S2OSC}

\subsection{Introduction of Forgetting}

In incremental OSC, we need to update model with the labeled instances of novel classes after detecting. Different from re-training with the entire previous in-class data, incremental model

\footnotetext{
${ }^{1}$ https://www.pythonware.com/products/pil/
} 
update aims to fine-tune the model only referring limited data from known classes. Therefore, the catastrophic forgetting phenomenon becomes an obstacle [4], i.e., we find that the knowledge learned from previous task (known classes classification) will lose when information relevant to the current task (novel class classification) is incorporated, considering the lack of previous in-class data.

\subsection{Model Update}

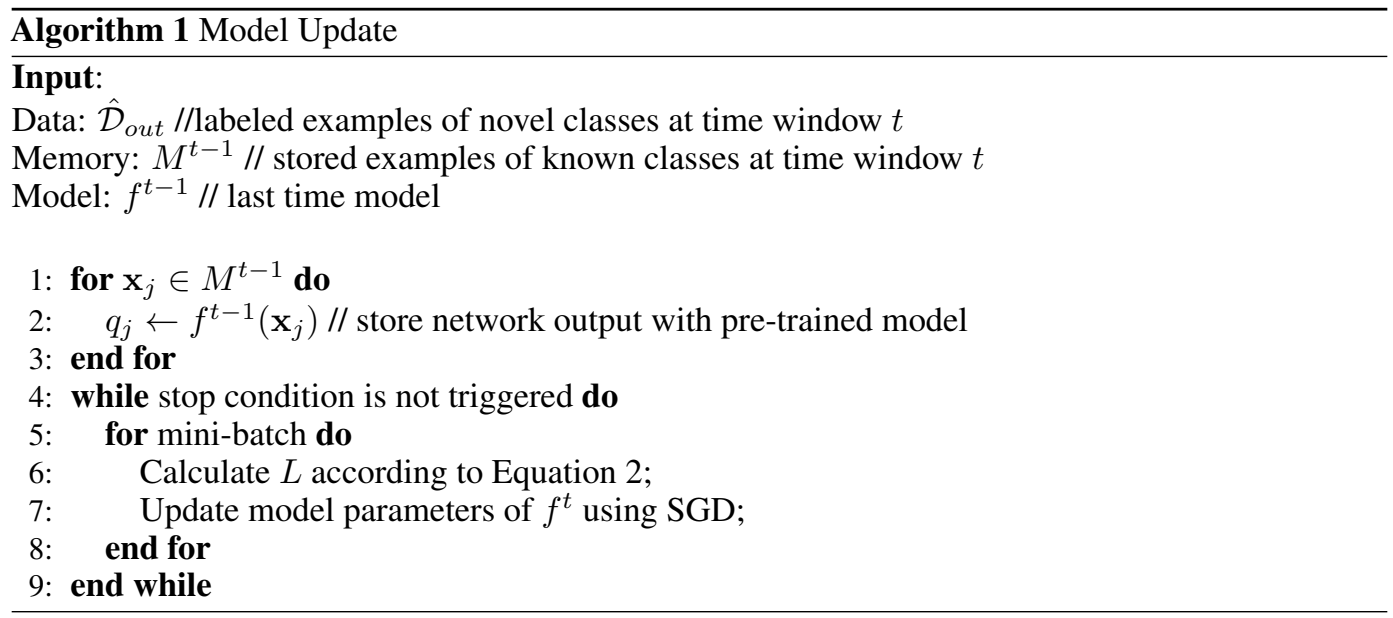

In this subsection, we will describe incremental model update in detail. After S2OSC operator, we can achieve potential out-of-class instances for querying their true labels. Suppose at time window $t$, we acquire $Q$ examples with ground-truths for novel classes, note that $Q \gg K$, where $K$ is the number of instances in data filtering. And these newly labeled examples constitute $\hat{\mathcal{D}}_{\text {out }}$. Therefore, the overall loss for fine-tuning $f$ can be relaxed as: $L=\ell\left(\hat{\mathcal{D}}_{\text {out }}, f^{t}\right)+\ell\left(M^{t-1}, f^{t}\right)$ according to the Eq. 3 in [5], which rephrases the constraint term as the task of better performance on $M^{t-1}$. This can be done with commonly used incremental update approach iCaLR [6]:

$$
\begin{aligned}
L & =-\left(\sum_{\substack{\mathbf{x}_{i} \in \hat{\mathcal{D}}_{\text {out }} \cup M^{t-1} \\
\mathbf{q}_{j}}}=f^{t-1}\left(\mathbf{x}_{j}\right)\right.
\end{aligned}
$$

Consequently, the loss function encourages the network to output the correct class indicator (classification loss) for all labeled examples, and reproduces the scores calculated in the previous step (distillation loss) for stored in-class examples. Besides, in the memory update phase, we reduce and add examples according to [6], which considers the structure representativeness. The details are shown in Algorithm 1.

Here, we adopt the replay-based methods as [6], rather than use regularization-based methods such as EWC [7], IMM [8] with extra regularization term on parameters to consolidate previous knowledge. The reason is that regularization-based methods always calculate fisher information matrix [7] for all parameters in network, which is hard to accomplish for convolutional neural networks. Note that EWC and IMM basically experiment with shallow fully connected network.

\section{Experiments}

\subsection{Datasets and Baselines}

In this subsection, we will give the details of datasets and baseline methods.

We utilize five publicly visual datasets for evaluating: FASHION-MNIST dataset contains 70,000 images of articles from 10 distinct classes, CIFAR-10 dataset includes 60,000 natural color images of $32 \times 32$ pixels from 10 different classes, SVHN dataset also includes 100,000 natural color images of $32 \times 32$ pixels about house numbers from 10 different classes, MNIST dataset contains 70,000 labeled handwritten digits images from 10 categories, CINIC scene dataset is enlarged CIFAR-10 with 
100,000 images. To validate the effectiveness on dataset with large classes, we further experiment on CIFAR-30 as [9], which randomly select 30 classes from CIFAR-100.

For baseline methods, we compare nine state-of-the-art methods including: 1) traditional outlier detection method: Iforest; 2) linear one-class OSC methods: One-Class SVM (One-SVM), LACUSVM (LACU) and SENC-MAS (SENC); 3) deep one-class OSC methods: ODIN-CNN (ODIN), CFO and CPE; and 4) deep multiple-class OSC methods: DTC. Abbreviations in parentheses. Specifically,

- Iforest: an ensemble tree method to detect outliers;

- One-Class SVM (One-SVM): a baseline for out-of-class detection and classification;

- LACU-SVM (LACU): a SVM-based method that incorporates the unlabeled data from open set for unknown class detection;

- SENC-MAS (SENC): a matrix sketching method that approximates original information with a dynamic low-dimensional structure;

- ODIN-CNN (ODIN): a CNN-based method that distinguishes in-distribution and out-ofdistribution over softmax score;

- CFO: a generative method that adopts an encoder-decoder GAN to generate synthetic unknown instances;

- CPE: a CNN-based ensemble method, which adaptively updates the prototype for detection;

- DTC: a extended deep transfer clustering method for novel class detection.

There are several instructions for baselines: 1) Iforest, ODIN and CFO can only perform binary classifications, i.e., whether the instance is an unknown class. Thus we further conduct unsupervised clustering on both know and unknown class data for subdividing; 2) all baselines are one-class methods except DTC, i.e., they also perform OSC in two steps: first detect the super-class of unknown classes, then perform unsupervised clustering; 3) all of baselines are OSC methods except LACU, SENC and CPE, but they can be applied in incremental OSC by combing memory data to update following [10], except Iforest which replies on the quality of clustering in current time window.

\subsection{Implementation}

We develop $f$ based on convolutional network structure ResNet34 [11], and $g$ based on ResNet18 [11]. Note that we use an identical set of hyperparameters $\left(\lambda=1, \alpha=0.3, \lambda_{u}=0.2, \tau=0.85\right.$, $T($ softmax $-T)=3, M=2000)$. In all of our models and experiments, we adopt standard SGD with Nesterov momentum [12], where the momentum $\beta=0.9$. We train the initial model $f$ as following: the number of epochs is 20 , the batch size is 64 , the learning rate is 0.01 , and weight decay is 0.001 . While training $g$ as following: the number of epochs is 30 , the batch size is 64 , the learning rate is 0.005 , and weight decay is 0.0005 . We implement all baselines and perform all experiments based on code released by corresponding authors. For CNN based methods, we use the same network architecture and parameters during training, such as optimizer, learning rate schedule, and data pre-processing. Our method is implemented on a Nvidia TITAN X GPU with Pytorch $0.4 .06^{2}$.

\subsection{Open Set Classification}

We additionally validate the effectiveness of S2OSC on various settings: 1) ablation study about S2OSC (section 4.3.1); 2) performance with different number of unknown classes (section 4.3.2); 3) case study of filtered out-of-class instances (section 4.3.3). Considering page limitation and representativeness of used datasets, we utilize three typical datasets, i.e., MNIST, CIFAR-10 and SVNH, for additional experiments.

\subsubsection{Ablation Study}

S2OSC has several variant designs. Therefore, in this subsection, we aim to analyze following questions: 1) Why choose the cross-entropy loss function for training $g$, without considering the large margin based loss function in traditional OSC methods [13, 10, 9], such as triplet loss [14],

\footnotetext{
${ }^{2}$ https://pytorch.org/
} 
Table 1: Ablation study about variants of S2OSC.

\begin{tabular}{l|c|c|c|c|c|c}
\hline \multirow{2}{*}{ Methods } & \multicolumn{3}{|c|}{ Accuracy } & \multicolumn{3}{c}{ F1 } \\
\cline { 2 - 8 } & MNIST & CIFAR-10 & SVHN & MNIST & CIFAR-10 & SVHN \\
\hline S2OSC-S & .834 & .812 & .748 & .792 & .771 & .666 \\
S2OSC-LM & .793 & .675 & .589 & .754 & .608 & .505 \\
S2OSC-FN & .891 & .817 & .880 & .846 & .777 & .834 \\
S2OSC-U & .886 & .786 & .812 & .853 & .813 & .833 \\
S2OSC & $\mathbf{. 9 8 5}$ & $\mathbf{. 8 4 7}$ & $\mathbf{. 8 9 8}$ & $\mathbf{. 9 8 5}$ & $\mathbf{. 8 5 4}$ & $\mathbf{. 9 0 1}$ \\
\hline Methods & \multicolumn{3}{|c|}{ Precision } & & & Recall \\
& MNIST & CIFAR-10 & SVHN & MNIST & CIFAR-10 & SVHN \\
\hline S2OSC-S & .758 & .736 & .602 & .834 & .812 & .748 \\
S2OSC-LM & .727 & .561 & .465 & .793 & .675 & .589 \\
S2OSC-FN & .807 & .746 & .795 & .891 & .817 & .880 \\
S2OSC-U & .765 & .758 & .876 & .786 & .886 & .812 \\
S2OSC & $\mathbf{. 8 4 7}$ & $\mathbf{. 9 7 2}$ & $\mathbf{. 8 8 8}$ & $\mathbf{. 7 9 9}$ & $\mathbf{. 9 8 6}$ & $\mathbf{. 9 8 5}$ \\
\hline
\end{tabular}

largin margin softmax [15]? 2) Why not directly perform semi-supervised learning based on the pre-trained model $f$ to obtain $g$, i.e., fine-tune the $f$ to acquire $g$ ? 3) Why not utilize only labeled data to calculate supervised loss for training $g$ ?

To answer the first question, traditional OSC methods adopted large margin loss to restrict intra-class and inter-class distance property, then detected novel class by identifying outliers. All of these approaches have a strong assumption that the embeddings or predictions learned by pre-trained model for out-of-class instances are apart from in-class ones. However, this assumption will fail on the data with complex content. Therefore, there is no need to use large margin loss here, and a unified classifier $f$ is more convenient for knowledge distillation in training $g$. More importantly, in our semi-supervised paradigm, for training classifier $g$, we first unify all unknown classes in $\mathcal{D}_{t e}$ as a super-class. If we utilize large margin loss function here, it will also reduce the intra-class distance of super-class, which further affect $g$ 's training considering semantic confusion and subsequent clustering operation.

As to the second question, the model $f$ is pre-trained with in-class data, but performing semisupervised re-training based on the pre-trained model $f$ has two limitations: 1) the number $K$ (examples in each class) of $\mathcal{X}$ is much smaller than that of $f$. Therefore, it is more inclined to classify the known classes and ignore the unknown classes if we fine-tune based on $f ; 2$ ) the $f$ can not be regarded as teacher network for knowledge distillation any more.

For last problem, we train $g$ in the semi-supervised manner as [1], which achieves the state-of-the-art classification performance comparing with other approaches. The number $K$ of examples in each class is limited, and thus supervised training may leads to overfitting.

To further verify our analysis, we conducted extra ablation experiments with the same setting as S2OSC on one unknown class: 1) S2OSC-S represents that we calculate loss $L_{u}$ including stronglyaugmented instances instead of weakly-augmented version; 2) S2OSC-LM denotes that S2OSC utilizes the common triplet loss to train $g$; 3) S2OSC-FN indicates that S2OSC directly fine-tunes based on $f$ to obtain $g$; 4) S2OSC-U represents that S2OSC removes the unsupervised term. Table 1 records the results, best results are in bold. We observe that $\mathrm{S} 2 \mathrm{OSC}$ outperforms all variant methods on three datasets with different criteria. This validate that: 1) the performances of strong augmentation is worse than weak augmentation, this indicates that the strong augmentation may introduce more noises which are difficult to train; 2) large-margin loss is not suitable for training $g$, even only with one unknown class; 3 ) fine-tuning base on $f$ is not a good choice, which validates the analysis above; 4) the contribution of unlabeled data is significant, this indicates that unlabeled data can enlarge the training data and contribute for the learning process.

\subsubsection{Influence of Unknown Class Number}

Considering that all datasets have 10 classes, thus we randomly hold out 5 classes as initial training data, and additionally tune the number of unknown classes in $\{3,5\}$ by randomly picking remaining 


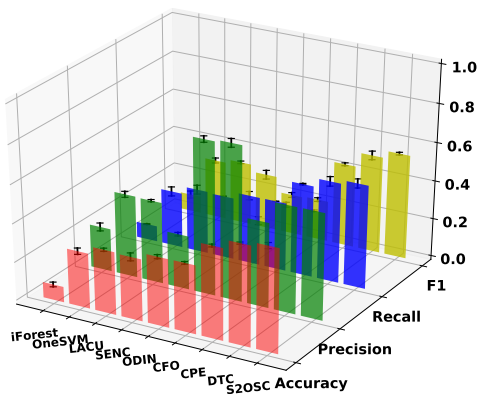

(a) MNIST-3

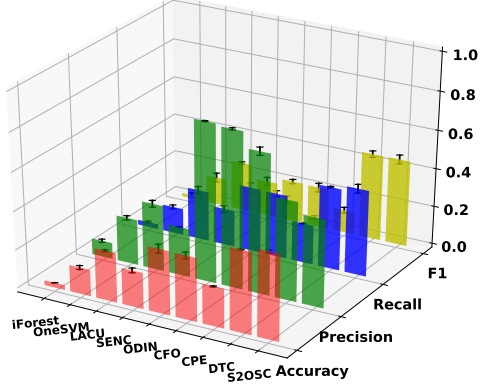

(c) CIFAR-3

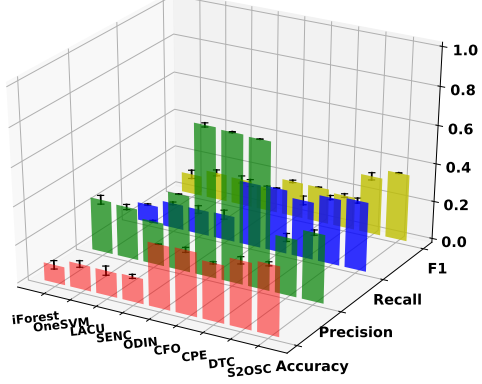

(e) SVNH-3

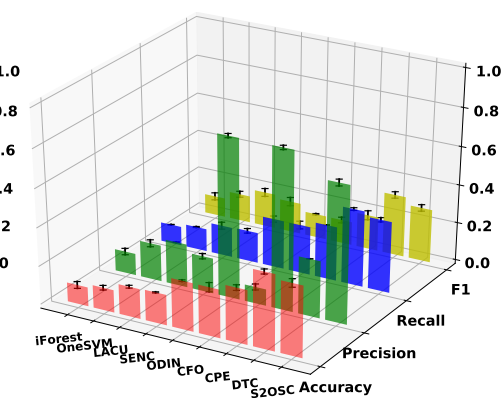

(b) MNIST-5

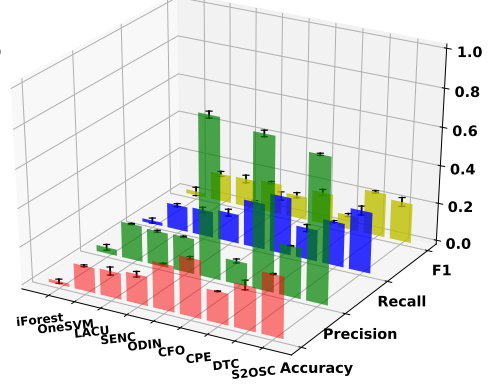

(d) CIFAR-5

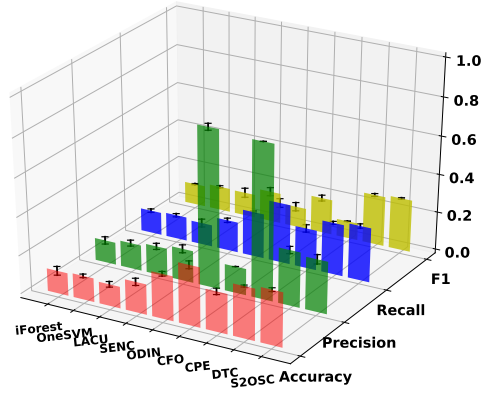

(f) SVNH-5

Figure 2: Classification performance of S2OSC and baseline methods on MNIST, CIFAR-10 and SVNH. Dataset- $p$ denotes with $p$ unknown classes. The X axis represents different OSC methods, the $\mathrm{Y}$ axis denotes the metrics, and the $\mathrm{Z}$ axis gives the performance.

classes (experiments about one unknown class have been reported, considering that our method is significantly superior to baselines, we did not report std). We also extract $33 \%$ of the known class data into test set, so that the test set mixes with known and unknown classes. The results report averaged performance over 5 random class partitions as [9].

Figure 2 records the experiment results (mean and std) of three typical datasets, which reveal that: 1) with the number of unknown classes increases, performance of all approaches decrease. This indicates that, once multiple unknown classes emerge in testing phase, the problem of embedding confusion will exacerbate, making OSC more complicated; 2) the precision of S2OSC is not high, whereas the recall of high precision model (e.g., CPE) is not very good, this indicates that most unknown classes are divided into known classes. The $\mathrm{F}_{\text {out }}$ of novel class detection in Table 2 also validates this phenomenon; 3) under three unknown classes scenario, S2OSC still outperforms all baselines on various criteria except recall. While S2OSC is competitive with other baselines under five unknown classes, i.e., S2OSC is lower than several baselines on MNIST dataset, and lower than CPE of precision on other two datasets.

Figure 3 shows feature embedding results using T-SNE [16]. The figures in upper row are T-SNE results with 3 unknown classes (i.e., 5, 6, 7), and figures in bottom row are with 5 unknown classes (i.e., 5, 6, 7, 8, 9, 10). Clearly, S2OSC can obviously distinguish between known and unknown 
Table 2: Comparison of novel class detection $\left(\mathrm{F}_{\text {out }}\right)$ with different number of unknown classes (SVM/Our denotes One-SVM/S2OSC).

\begin{tabular}{|c|c|c|c|c|c|c|c|c|c|}
\hline & \multicolumn{3}{|c|}{ One Class } & \multicolumn{3}{|c|}{ Three Classes } & \multicolumn{3}{|c|}{ Five Classes } \\
\hline & MNIST & CIFAR & SVHN & MNIST & CIFAR & SVHN & MNIST & CIFAR & SVHN \\
\hline Iforest & $.065 \pm .010$ & $.251 \pm .032$ & $.230 \pm$ & $.292 \pm .047$ & $.139 \pm$ & $.224 \pm .037$ & $.407 \pm .060$ & $.224 \pm .031$ & $219=$ \\
\hline VI & & & .300 & .494 & & & & & \\
\hline $\mathrm{LACU}$ & $.223 \pm .026$ & $.190 \pm .009$ & $.251 \pm .018$ & $.381 \pm .058$ & $.449 \pm .026$ & $.465 \pm .102$ & $.514 \pm .067$ & $.604 \pm .036$ & $.594 \pm .019$ \\
\hline SENC & $.005 \pm .000$ & $.110 \pm .003$ & $.007 \pm 0$ & $.530 \pm .002$ & $.554 \pm .040$ & $.472 \pm .075$ & $.690 \pm .004$ & $.689 \pm .003$ & $.706 \pm .018$ \\
\hline DIN & $.390 \pm .085$ & $.031 \pm$ & $.219=$ & $.006 \pm 0$ & $.006 \pm$ & $.006 \pm$ & .13 & $.130 \pm 0$ & $.130 \pm$ \\
\hline CFO & .27 & $.275 \pm$ & $.285 \pm$ & $.296=$ & $.442 \pm$. & $.452 \pm$ & .543 & $.556 \pm .010$ & $.596 \pm .072$ \\
\hline CPE & $.923 \pm .015$ & $.383 \pm .022$ & $.563 \pm .053$ & $.424 \pm .055$ & $.385 \pm 0.026$ & $.338 \pm .094$ & $.619 \pm .040$ & $.557 \pm .050$ & $.577 \pm .119$ \\
\hline DTC & $.249 \pm .041$ & $.252 \pm .028$ & $.142 \pm .016$ & $.074 \pm .009$ & $.241 \pm 0.176$ & $.309 \pm .084$ & $.553 \pm .131$ & $.593 \pm .002$ & $.756 \pm .055$ \\
\hline Our & $.987 \pm .019$ & $.853 \pm .083$ & $.932 \pm .032$ & $.629 \pm 013$ & $.632 \pm 0.024$ & $.666 \pm .117$ & $.974 \pm .001$ & $.858 \pm .028$ & $.830 \pm .029$ \\
\hline
\end{tabular}

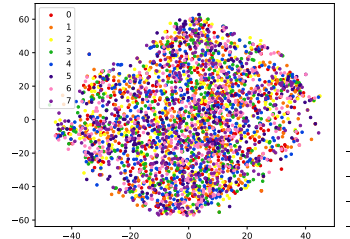

(a-1) Original-3

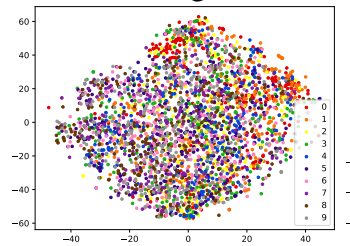

(a-2) Original-5

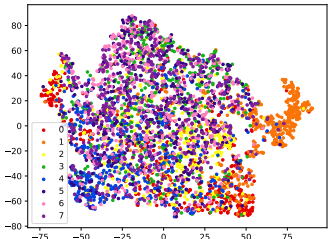

$(b-1)$ CPE-3

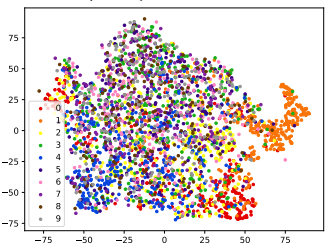

$(b-2)$ CPE-5

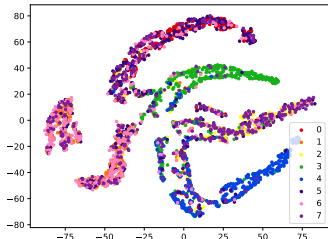

$(c-1)$ CFO-3

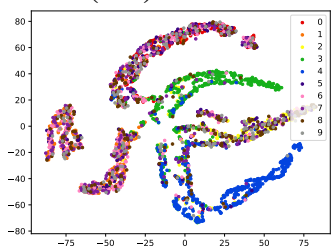

$(c-2) \mathrm{CFO}-5$

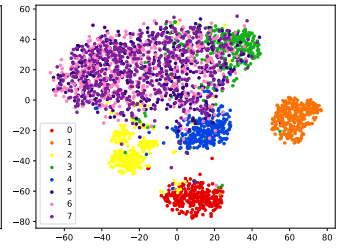

$(d-1)$ S2OSC-3

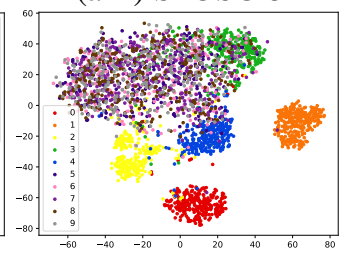

$(d-2)$ S2OSC-5

Figure 3: T-SNE Visualization on CIFAR-10 dataset. Method- $p$ denotes with $p$ unknown classes. (a) original feature space; (b) learned representations by discriminative method CPE; (c) learned representations by generative detection method CFO; (d) learned representations by proposed S2OSC.

classes, i.e., instances from unknown classes are well separated from known clusters comparing with other baselines, which benefits unknown class detection in result.

For further measuring the discrimination of known and unknown classes, we utilize another criterion in [10], which treats OSC as a binary classification problem placing emphasis on novel class detection. In detail, we consider all known classes as negative and all unknown classes as positive. $F_{\text {out }}=$ $\frac{2 T P}{2 T P+F P+F N}$ is $\mathrm{F} 1$ of unknown classes, $T P, F P, F N, T N$ denotes the true positives, false positive, false negatives and true negatives. Table 2 gives the results, best results are in bold. We can observe that novel class detection, i.e., F1 indicator, of S2OSC is significantly higher than other methods on various settings and ODIN is difficult to handle multiple unknown classes. An interesting phenomenon is that the F1 of three unknown classes is lower than that of one and five unknown classes. This is because one unknown class does not have the problem of intra-class confusion existing in multiple unknown classes, thus has better performance. On the other hand, with the number of unknown classes increasing, the embedding confused instances in filtered data decrease, thus it is more conducive to the training $g$ and improves performance.

\subsubsection{Case Study}

We also give some examples of distinct and confused instances for display. Here we consider one unknown class case on CIFAR-10 dataset, in which the known class set includes: "airplane, cat, deer, automobile, truck", and the unknown class is "dog". Then we sort $\mathcal{D}_{t e}$ according to the weights $w$, and select instances according to the ranking. As shown in Figure 4, we observe that: 1) most of the distinct instances are dogs, but still include few known class data, for example, instances from cat. It can be seen from the examples that many confused cats are outliers, which are difficult to distinguish; 


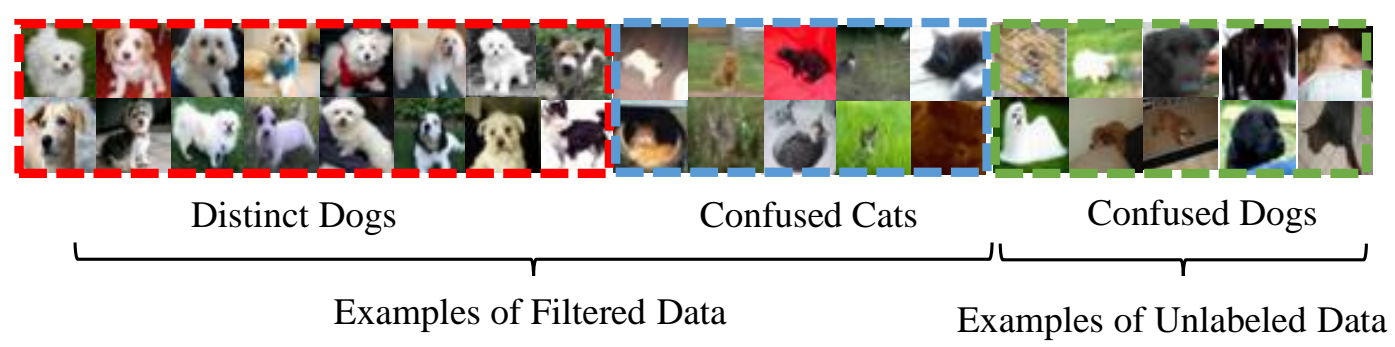

Figure 4: Examples of filtered instances with large weights $w$ and unselected out-of-class instances by proposed S2OSC.

2) most distinct dogs have more diagnostic characteristics, for example, the images with full-body shot; 3 ) unselected dogs are ambiguous, for example, dogs with only head or unclear dog images. Thus, combining labeled data into unlabeled loss is helpful for training $g$.

\subsubsection{Large-Scale OSC}

To validate the effectiveness of our proposed method on large-scale OSC setting, i.e., dataset with large-scale classes. We conduct more experiments on CIFAR-30 following [9]. In detail, we adopt the split class case following [9], which holds out 10 classes as out-of-class for testing, and leaves the remaining classes as the initial training set. The experimental setup is same as the case of multiple unknown classes. Table 3 records the results, best results are in bold. We can observe that: 1) the performances of all methods decrease rapidly facing large-scale OSC; 2 ) we have similar results as other setups that S2OSC consistently outperforms all baselines on various criteria except F1, which ranks runner-up. For example, S2OSC provides at least $10 \%$ improvements of accuracy than other baselines. This shows that S2OSC can well perform OSC on different class scales.

Table 3: Comparison of large-scale open set classification with multiple unknown classes.

\begin{tabular}{l|c|c|c|c|c|c|c|c|c}
\hline Methods & Iforest & One-SVM & LACU & SENC & ODIN & CFO & CPE & DTC & S2OSC \\
\hline Accuracy & .006 & .009 & .008 & .007 & .163 & .147 & .176 & .181 & $\mathbf{. 3 0 2}$ \\
Precision & .709 & .703 & .195 & .179 & .340 & .436 & .245 & .248 & $\mathbf{. 7 9 1}$ \\
Recall & .006 & .009 & .008 & .007 & .163 & .147 & .176 & .181 & $\mathbf{. 3 0 2}$ \\
F1 & .006 & .008 & .008 & .007 & .147 & .125 & .165 & $\mathbf{. 1 6 8}$ & .165 \\
F $_{\text {out }}$ & .649 & .614 & .691 & .706 & .917 & .898 & .917 & .917 & $\mathbf{. 9 4 0}$ \\
\hline
\end{tabular}

\subsection{Incremental Open Set Classification}

\subsubsection{Streaming Dataset}

In this subsection, we mainly give the details of streaming data construction and measure criteria. In this paper, we perform incremental OSC, rather than online OSC, thus we need to accept the testing data before performing unknown class detection. There exists many related scenarios, such as sequential task learning in lifelong learning.

We consider single novel class case here following $[13,10,9]$. In detail, for each dataset, we randomly choose $50 \%$ from the total classes as known class set, the rests are regarded as unknown class set. The data of known class set can be divided into two parts: 1) $50 \%$ of data are regarded as initial training data; 2) the remaining data are used to constitute a streaming data. We simulate a streaming data as shown in Figure 5 (a). The data before time $t_{0}$ are training data. Then each class simulates an independent streaming data by shuffling instances randomly and arranging the data according to the index. A new class of streaming data appends every fixed time interval $\Delta t$. Thus, the instances occur in $\Delta t$ constitute a time window data mixed with known and unknown instances.

For calculating Forgetting criterion, let $a c c_{k, j}$ be the accuracy evaluated on the known class set, i.e. the data of classes emerging on $j$-th time window $(j \leq k)$, after training the network incrementally from stage 1 to $k$, the average accuracy at time $k$ is defined as: $A_{k}=\frac{1}{k} \sum_{j=1}^{k} a c c_{k, j}$ [18]. Higher $A_{k}$ 
represents better classifier. Thus, to validate the catastrophic forgetting, we calculate the performance on forgetting profile as [18], i.e., Forgetting $=\frac{A^{*}-\text { mean }(A)}{A^{*}}, A^{*}$ is the optimal accuracy with entire data, $A$ is the set of average accuracy.

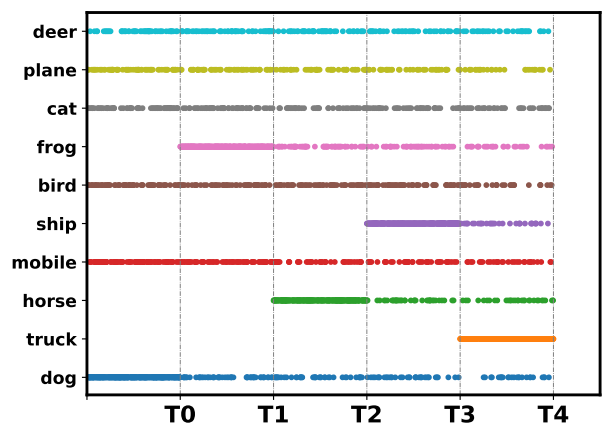

(a) single unknown class

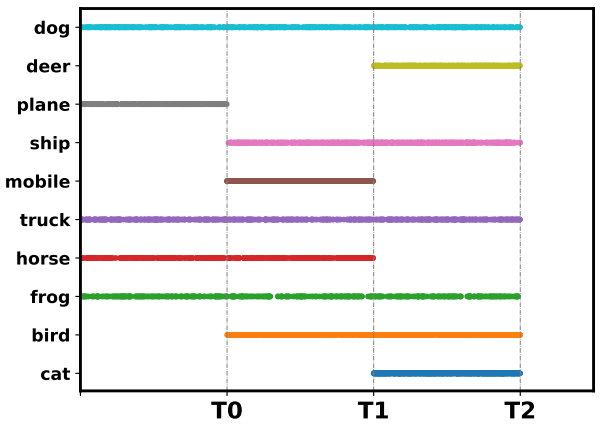

(b) multiple unknown classes

Figure 5: The class distribution of simulated streams on CIFAR-10 dataset. The X-axis denotes time scale and Y-axis is class information. (a) is streaming data with single unknown class, (b) denotes streaming data with multiple unknown classes. Note that some known classes may disappear in time window $t$ in figure (b) in order to be more in line with real applications.

\subsubsection{Incremental Multi-Class Case}

In order to be more in line with the real scene, we consider additional case of multiple unknown classes here. The main difference from single unknown class is that more than one unknown class may appear in each time window. To solve this problem, considering that all datasets are with 10 classes, we firstly select 5 classes into known class set, the rest 5 classes are regarded as unknown class set. Then, we randomly select a number $l$ from 1-5 as the number of time windows, and randomly divide the unknown class set into $l$ parts. In result, we can obtain a incremental class order that appears in each time window. The generation of streaming data is the same as that of single unknown class setting. A case of generated streaming data of CIFAR-10 is shown in Figure 5 (b).

We only give the results of CIFAR-10 which is with complex content here. Table 4 compares the classification and forgetting performance of I-S2OSC with all baselines over streaming data under multiple novel classes case. Best results are in bold, N/A denotes no result. We can observe similar results as single novel class setting that I-S2OSC consistently outperforms all baselines on various criteria and has the least forgetting for update. We further compare the run time of I-S2OSC with other deep baselines. Considering the superior performance and model comparability, we only compare with CPE and DTC here. Specifically, the run time of I-S2OSC/CPE/DTC is 1.033/1.583/1.8 hours, we can observe that the run time of I-S2OSC is significantly less than other methods, because CPE and DTC employ losses based on embeddings, which convergences slowly.

Table 4: Comparison of incremental open set classification with multiple unknown classes.

\begin{tabular}{l|c|c|c|c|c|c|c|c|c}
\hline Methods & Iforest & One-SVM & LACU & SENC & ODIN & CFO & CPE & DTC & I-S2OSC \\
\hline Accuracy & .106 & .186 & .175 & .220 & .333 & .315 & .314 & .356 & $\mathbf{. 4 4 4}$ \\
Precision & .257 & .296 & .281 & .381 & .473 & .508 & .471 & .471 & $\mathbf{. 6 0 6}$ \\
Recall & .106 & .186 & .175 & .220 & .333 & .315 & .356 & .356 & $\mathbf{. 4 4 2}$ \\
F1 & .132 & .205 & .194 & .134 & .271 & .259 & .399 & .399 & $\mathbf{. 4 8 7}$ \\
F $_{\text {out }}$ & .230 & .406 & .404 & .685 & .796 & .736 & .233 & .233 & $\mathbf{. 8 0 2}$ \\
Forgetting & N/A & .625 & .633 & .678 & .606 & .390 & .374 & .374 & $\mathbf{. 3 0 5}$ \\
\hline
\end{tabular}

\section{Broader Impact}

In this work, we study the problem of open set classification and incremental extension. We are aware that these technique may pose ethical issues, for example, people or institutions may use this 
technology to judge the efficiency of the system on detecting novel illegal pictures. Although there exist uncertainty within the technique, we consider that it is necessary to research and develop such techniques for understanding the dynamic environment. More reliable controls are expected from further discussion of these techniques.

\section{References}

[1] Sohn, K., D. Berthelot, C. Li, et al. Fixmatch: Simplifying semi-supervised learning with consistency and confidence. CoRR, abs/2001.07685, 2020.

[2] Berthelot, D., N. Carlini, E. D. Cubuk, et al. Remixmatch: Semi-supervised learning with distribution matching and augmentation anchoring. In ICLR. 2020.

[3] Cubuk, E. D., B. Zoph, D. Mane, et al. Autoaugment: Learning augmentation strategies from data. In CVPR, pages 113-123. 2019.

[4] Ratcliff, R. Connectionist models of recognition memory: constraints imposed by learning and forgetting functions. Psychol. Review, 97(2):285, 1990.

[5] Song, G., X. Tan. Sequential learning for cross-modal retrieval. In ICCV Workshop, pages 4531-4539. 2019.

[6] Rebuffi, S.-A., A. Kolesnikov, G. Sperl, et al. icarl: Incremental classifier and representation learning. In CVPR, pages 5533-5542. 2017.

[7] Kirkpatrick, J., R. Pascanu, N. C. Rabinowitz, et al. Overcoming catastrophic forgetting in neural networks. CoRR, abs/1612.00796, 2016.

[8] Lee, S.-W., J.-H. Kim, J. Jun, et al. Overcoming catastrophic forgetting by incremental moment matching. In NIPS, pages 4655-4665. 2017.

[9] Geng, C., S. Huang, S. Chen. Recent advances in open set recognition: A survey. CoRR, abs/1811.08581, 2018.

[10] Wang, Z., Z. Kong, S. Chandra, et al. Robust high dimensional stream classification with novel class detection. In ICDE, pages 1418-1429. 2019.

[11] He, K., X. Zhang, S. Ren, et al. Deep residual learning for image recognition. In CVPR, pages 770-778. 2016.

[12] Sutskever, I., J. Martens, G. E. Dahl, et al. On the importance of initialization and momentum in deep learning. In ICML, pages 1139-1147. 2013.

[13] Da, Q., Y. Yu, Z.-H. Zhou. Learning with augmented class by exploiting unlabeled data. In AAAI, pages 1760-1766. 2014.

[14] Kulis, B. Metric learning: A survey. Foundations and Trends in Machine Learning, 5(4):287-364, 2013.

[15] Liu, W., Y. Wen, Z. Yu, et al. Large-margin softmax loss for convolutional neural networks. In ICML, pages 507-516. 2016.

[16] Maaten, L. v. d., G. Hinton. Visualizing data using t-sne. Journal of machine learning research, 9(Nov):2579-2605, 2008.

[17] Han, K., A. Vedaldi, A. Zisserman. Learning to discover novel visual categories via deep transfer clustering. In ICCV, pages 8400-8408. 2019.

[18] Chaudhry, A., P. K. Dokania, T. Ajanthan, et al. Riemannian walk for incremental learning: Understanding forgetting and intransigence. CoRR, abs/1801.10112, 2018. 